\title{
Comparison between urine albumin-to- creatinine ratio and urine protein dipstick testing for prevalence and ability to predict the risk for chronic kidney disease in the general population (Iwate-KENCO study): a prospective community-based cohort study
}

Yorihiko Koeda" ${ }^{*}$, Fumitaka Tanaka', Toshie Segawa ${ }^{1}$, Mutsuko Ohta², Masaki Ohsawa ${ }^{3}$, Kozo Tanno $^{3}$, Shinji Makita', Yasuhiro Ishibashi ${ }^{1}$, Kazuyoshi Itai ${ }^{3}$, Shin-ichi Omama ${ }^{4}$, Toshiyuki Onoda ${ }^{3}$, Kiyomi Sakata ${ }^{3}$, Kuniaki Ogasawara ${ }^{4}$, Akira Okayama $^{5}$ and Motoyuki Nakamura ${ }^{1}$

\begin{abstract}
Background: This study compared the combination of estimated glomerular filtration rate (eGFR) and urine albumin-to-creatinine ratio (UACR) vs. eGFR and urine protein reagent strip testing to determine chronic kidney disease (CKD) prevalence, and each method's ability to predict the risk for cardiovascular events in the general Japanese population.
\end{abstract}

Methods: Baseline data including eGFR, UACR, and urine dipstick tests were obtained from the general population ( $n=22$ 975). Dipstick test results (negative, trace, positive) were allocated to three levels of UACR $(<30,30-300,>300)$, respectively. In accordance with Kidney Disease Improving Global Outcomes CKD prognosis heat mapping, the cohort was classified into four risk grades (green: grade 1; yellow: grade 2; orange: grade 3, red: grade 4) based on baseline eGFR and UACR levels or dipstick tests.

Results: During the mean follow-up period of 5.6 years, 708 new onset cardiovascular events were recorded. For CKD identified by eGFR and dipstick testing (dipstick test $\geq$ trace and eGFR $<60 \mathrm{~mL} / \mathrm{min} / 1.73 \mathrm{~m}^{2}$ ), the incidence of CKD was found to be $9 \%$ in the general population. In comparison to non-CKD (grade 1), although cardiovascular risk was significantly higher in risk grades $\geq 3$ (relative risk $(R R)=1.70 ; 95 \% \mathrm{Cl}: 1.28-2.26$ ), risk predictive ability was not significant in risk grade $2(R R=1.20 ; 95 \% \mathrm{Cl}: 0.95-1.52)$. When CKD was defined by eGFR and UACR (UACR $\geq 30 \mathrm{mg} / \mathrm{g}$ $\mathrm{Cr}$ and eGFR $\left.<60 \mathrm{~mL} / \mathrm{min} / 1.73 \mathrm{~m}^{2}\right)$, prevalence was found to be $29 \%$. Predictive ability in risk grade $2(R R=1.41 ; 95 \%$ Cl: 1.19-1.66) and risk grade $\geq 3(R R=1.76 ; 95 \%$ Cl: 1.37-2.28) were both significantly greater than for non-CKD. Reclassification analysis showed a significant improvement in risk predictive abilities when CKD risk grading was based on UACR rather than on dipstick testing in this population $(p<0.001)$.

Conclusions: Although prevalence of CKD was higher when detected by UACR rather than urine dipstick testing, the predictive ability for cardiovascular events from UACR-based risk grading was superior to that of dipstick-based risk grading in the general population.

Keywords: Cardiovascular disease, Renal function, Risk factor, Urine albumin-to-creatinine ratio, Urine dipstick test

\footnotetext{
*Correspondence: ori-aka@hotmail.co.jp

'Division of Cardiology, Department of Internal Medicine, Iwate Medical

University, 19-1 Uchimaru, Morioka, Iwate 020-8505, Japan

Full list of author information is available at the end of the article
} 


\section{Background}

Since the National Kidney Foundation began drawing attention to chronic kidney disease (CKD), several studies have reported that $\mathrm{CKD}$ is an independent risk factor for cardiovascular disease and cardiovascular mortality $[1,2]$. For people with CKD, the risk for death from a cardiovascular event is up to 20 times greater than the risk for requiring dialysis or transplantation [3]. A recent metaanalysis obtained from 1.5 million inhabitants in mainly Western populations reported that albuminuria levels are important for evaluating overall risk for CKD independent of estimated glomerular filtration rate (eGFR) [4]. In accordance with Kidney Disease Improving Global Outcomes (KDIGO) recommendations, several clinical practice guidelines in Europe, Australia, and Japan have recommended that prognostic grading for CKD should be based on a combination of urine albumin levels and eGFR [5-7].

Measurement of urine albumin, however, is not easily performed in clinical and screening settings, as it is expensive, creates a delay in the availability of results, and must be performed by laboratory technicians. Accordingly, despite relatively limited evidence for its clinical utility, urine dipstick testing remains a popular tool in epidemiological surveys.

To date, few reports have described the relationship between urine albumin-to-creatinine ratio (UACR) and urine protein reagent strip testing in terms of utility for CKD definition and risk grading in the general population. Specifically, it is not yet known how much the characteristics, prevalence, and risk predictive abilities for cardiovascular events of CKD change when UACR is used rather than dipstick testing for detection of this condition. In the current study, we examined the value of using UACR rather than urine dipstick testing to determine CKD prevalence, and compared each method's ability to predict the risk for cardiovascular events in the general population.

\section{Methods}

\section{Study participants}

This study was a prospective community-based cohort study examining cardiovascular events in Iwate Prefecture in northern Honshu, Japan. A total of 26469 residents in Ninohe, Kuji, and Miyako consented to participate in the study. All participants provided written informed consent. The study was part of the IwateKENCO (Kenpoku Cohort) study, as described previously [8-10].

The baseline survey was conducted between 2002 and 2004 and comprised a self-administered lifestyle questionnaire, blood pressure measurements, anthropometrical measurements, blood collection, and random spot urine sampling. A total of 22975 participants were enrolled who had complete data for eGFR, UACR, and dipstick urinalysis for proteinuria with no past history of stroke or myocardial infarction (7 841 men and 15134 women, aged 40-89 years, mean age of 62.9 years).

The study protocol was approved by the Ethics Committee of Iwate Medical University and conducted in accordance with the principles contained in the Declaration of Helsinki.

Blood pressure was measured twice using a Nippon Colin BP-103i II blood pressure monitor (model 513000; Nippon Colin, Komaki, Japan), with the mean value being used for statistical analysis. Peripheral venous blood samples were taken from the upper arm with participants in a seated position after at least 5 min rest. Blood samples were centrifuged immediately after collection and used for measurements. Creatinine $(\mathrm{Cr})$ concentrations in urine and serum were measured by the enzymatic method using a Hitachi 7700 automatic analyzer with standardized calibrators (Hitachi, Tokyo, Japan), and urine albumin measurement was by immunonephelometry (N-antiserum to human albumin assay; Dade Behring, Tokyo, Japan). Urine albumin concentrations were corrected to urine $\mathrm{Cr}$ concentrations and expressed as UACR $\mathrm{mg} / \mathrm{g} \mathrm{Cr}$. Urine protein levels were semiquantified using a Hema Combistix urine protein reagent testing strip (Siemens Healthcare Diagnostics, Tokyo, Japan). Results of the urine dipstick test were visually interpreted by trained laboratory technicians and recorded as $(-)$, trace, $(1+),(2+)$, or $(3+)$. Data from women who said they were menstruating were excluded in the study, as the urine test might be affected by hemoglobin in urine. eGFR was calculated using the formula devised by the Chronic Kidney Disease Epidemiology Collaboration (CKD-EPI) [11].

Following practice guidelines developed by the KDIGO proposal [12], participants were divided into an 18-part matrix comprising six categories of eGFR $(\geq 90,60-89$, $45-59,30-44,15-29,<15 \mathrm{~mL} / \mathrm{min} / 1.73 \mathrm{~m}^{2}$ ) across three categories of UACR $(<30,30-300,>300)$ or dipstick test (negative, trace, positive) as shown in Fig. 1. The reliability of the allocation of dipstick urinalysis (negative, trace, positive) to three levels of UACR $(<30,30-300,>300)$ has been reported in previous studies $[13,14]$. Accordingly,

\begin{tabular}{|ccccc}
\hline & $\begin{array}{c}\text { Proteinuria } \\
\text { (dipstick) }\end{array}$ & $(-)$ & $(+/-)$ & $>=(+)$ \\
\hline $\begin{array}{c}\text { UACR } \\
(\mathrm{mg} / \mathrm{g} \cdot \mathrm{Cr})\end{array}$ & $<29$ & $30-299$ & $>=300$ \\
\hline & $>=90$ & Grade 1 & Grade 2 & Grade 3 \\
& $60-89$ & Grade 2 & Grade 3 & \\
$\begin{array}{c}\text { GFR } \\
(\mathrm{mL} / \mathrm{min} /\end{array}$ & $45-59$ & Grade 3 & & \\
$\left.1.73 \mathrm{~m}^{2}\right)$ & $30-44$ & Grade 4 & \\
& $15-29$ & & & \\
& $<15$ & & & \\
\hline
\end{tabular}

Fig. 1 Modified KDIGO risk grading for CKD (six rows $\times$ three columns $=18$ matrix) 
the definition of CKD was two ways (the dipstick-based definition = dipstick test $\geq$ trace and eGFR $<60 \mathrm{~mL} / \mathrm{min} /$ $1.73 \mathrm{~m}^{2}$ : the UACR-based definition $=\mathrm{UACR} \geq 30 \mathrm{mg} / \mathrm{g} \mathrm{Cr}$ and eGFR $<60 \mathrm{~mL} / \mathrm{min} / 1.73 \mathrm{~m}^{2}$ ) Participants were then classified into four risk grades based on the matrix as shown in the heat map for both definitions (Fig. 1) (green: non-CKD (grade 1); yellow: moderately increased risk (grade 2); orange: high risk (grade 3); red: very high risk (grade 4)).

Diabetes mellitus was defined as a hemoglobin A1c (HbA1c) level $\geq 6.5 \%$, fasting blood glucose level $\geq 126 \mathrm{mg} / \mathrm{dL}$, blood glucose $\geq 200 \mathrm{mg} / \mathrm{dL}$, or undergoing treatment with antidiabetic drugs including insulin. Hypertension was defined as systolic blood pressure $\geq 140 \mathrm{mmHg}$, diastolic blood pressure $\geq 90 \mathrm{mmHg}$, or undergoing treatment with antihypertensive drugs. Hyperlipidemia was defined as total cholesterol $\geq 240 \mathrm{mmHg}$ or undergoing treatment with antihyperlipidemic drugs. Obesity was defined as body mass index (BMI) $\geq 25$.

\section{Endpoint}

The endpoint was determined as the composite of cardiovascular events (stroke, myocardial infarction, and sudden cardiac death occurring within $24 \mathrm{~h}$ of the onset of acute symptoms and signs). The incidence of hospitalized myocardial infarction was registered according to criteria of the MONICA study [15]. The study team visited all referral hospitals within the study area and reviewed medical charts or discharge summaries of almost all admitted patients. In cases where clinical data met the definition, the patient's data were registered. Stroke events were identified by accessing the Iwate Prefecture stroke registration program, which included the entire area where participants lived; details have been described previously [16, 17]. The follow-up survey for acute myocardial infarction and stroke was carried out from the date of the baseline survey until March 2009. The research methods have been described in detail previously $[8-10,17]$.

\section{Statistical analysis}

Continuous variables are expressed as mean \pm SD. Comparisons among CKD risk grades were conducted using one way analysis of variance or chi-squared tests. The Cox proportional hazards model was used to analyze the relative risk (RR) for cardiovascular events in each risk grade adjusted by several cofounding factors (age, sex, hypertension, hyperlipidemia, diabetes mellitus, current smoking, BMI, and atrial fibrillation). To determine concordance between two types of urine protein levels on a dichotomous outcome of UACR $(<30,30-300,>300)$ and the urine dipstick test (negative, trace, positive), the kappa statistic was calculated. Analyses were performed using the SPSS 20.0 for Windows. Testing the utility of
CKD risk grading employing UACR rather than dipstick testing was performed by reclassification tables and tested by Net Reclassification Improvement (NRI) and Integrated Discrimination Improvement (IDI) using the R 3.0.2 software package (www.r-project.org).

\section{Results}

\section{Clinical characteristics and prevalence}

Table 1 shows the clinical backgrounds of the study participants. The average eGFR value for the entire cohort was $76.8 \mathrm{~mL} / \mathrm{min} / 1.73 \mathrm{~m}^{2}$. Seven hundred and eight participants experienced a first onset of a cardiovascular event during the follow-up period (myocardial infarction or sudden cardiac death: $n=145$; stroke: $n=563$ ).

As shown in Fig. 2 (left), the dipstick-based definition of CKD (dipstick test $\geq$ trace and eGFR $<60 \mathrm{~mL} / \mathrm{min}$ / $1.73 \mathrm{~m}^{2}$ ) showed the prevalence of CKD in the general population as $9 \%$ (men $12 \%$, women $7 \%$ ) and prevalence rates of $91 \%$ for risk grade 1 (green), $6 \%$ for grade 2 (yellow), $2 \%$ for grade 3 (orange), and $1 \%$ for grade 4 (red). As shown in Fig. 2 (right), the UACR-based definition (UACR $\geq 30 \mathrm{mg} / \mathrm{g} \mathrm{Cr}$ and eGFR $<60 \mathrm{~mL} / \mathrm{min} /$ $1.73 \mathrm{~m}^{2}$ ) showed the prevalence of CKD as $29 \%$ in the general population (men $29 \%$, women $28 \%$ ), with proportions of $71 \%$ in risk grade 1 (green), $25 \%$ in grade 2 (yellow), $3 \%$ in grade 3 (orange), and $1 \%$ in grade 4 (red). The prevalence of CKD according to the UACRbased definition was nearly three times that of the dipstick-based definition (9\% compared with $29 \%$ ).

For both methods of CKD assessment, age, BMI, and percentages for diabetes mellitus, hypertension, and atrial fibrillation rose significantly with increasing progression of risk grade, but percentages for hyperlipidemia and current smoking did not differ across risk grades (Table 1).

For dipstick-based risk grades, $60 \%$ of CKD cases showed a negative indication for proteinuria. This result suggests that this method for CKD assessment was mainly based on decreased eGFR $\left(<60 \mathrm{~mL} / \mathrm{min} / 1.73 \mathrm{~m}^{2}\right)$ with no increase in urine protein levels. In contrast, $79 \%$ of UACR-based CKD cases had elevated UACR levels $(\geq 30 \mathrm{mg} / \mathrm{g} \mathrm{Cr}$ ) without reduced eGFR.

\section{Relationship between UACR and dipstick testing}

To examine the relationship between UACR levels $(<30$, $30-300,>300)$ and semi-quantitative dipstick test results (negative, trace, positive), a multiple comparison graph was constructed (Fig. 3). Even when dipstick urinalysis was negative, $22 \%$ of participants had UACR $\geq 30 \mathrm{mg} / \mathrm{g}$ $\mathrm{Cr}$. When the degree of coincidence between UACR levels $(<30,30-300,>300)$ and dipstick results (negative, trace, positive) was tested using the kappa statistic, the coefficient was relatively low (0.171). Sensitivity of the 
Table 1 Baseline characteristics among CKD risk grades in the general population

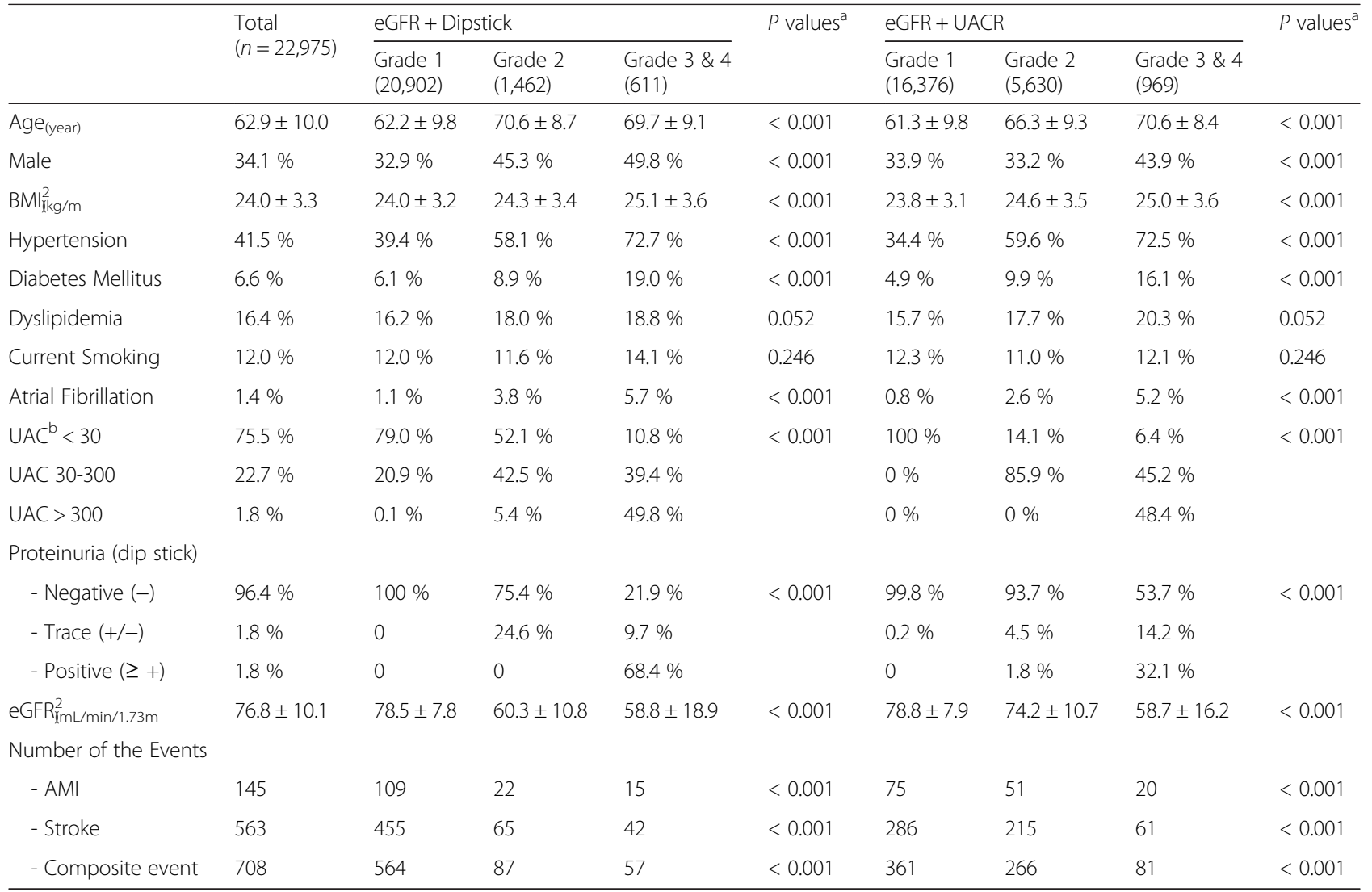

a Differences among the CKD grades

b Urine Albumin Creatinine Ratio

urine dipstick test $(\geq+1)$ for UACR (more than $30 \mathrm{mg} / \mathrm{g} \mathrm{Cr}$ ) was $8 \%$, and specificity was $100 \%$.

\section{Risk grades and cardiovascular events}

When compared with the non-CKD group (risk grade 1), RR for cardiovascular events derived from a Cox proportional hazards model was 1.36 (95\% CI: 1.12-1.65) for dipstick-based CKD, and 1.47 (95 \% CI: 1.25-1.72) for UACR-based CKD. Both methods of CKD assessment on the whole showed similar predictive abilities for

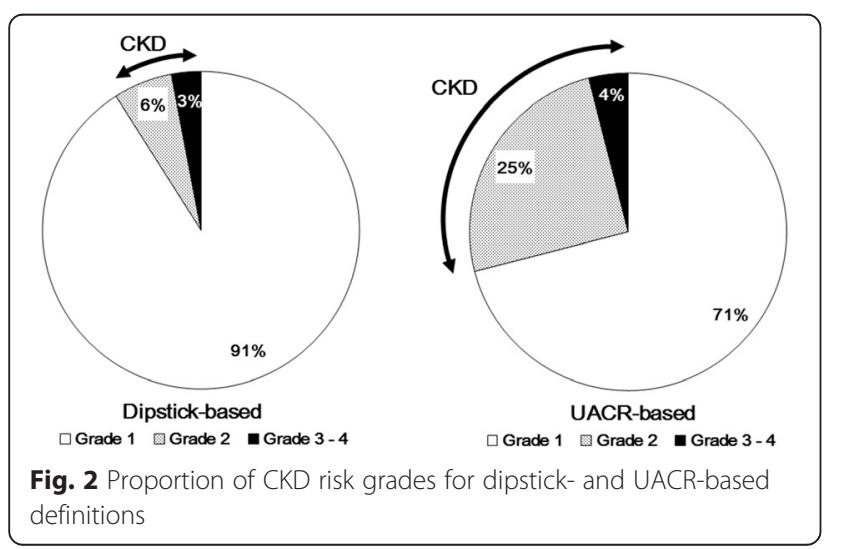

cardiovascular events. When the cohort was divided into four risk grades based on urine dipstick testing, RR for cardiovascular events were 1.20 (95 \% CI: 0.95-1.52) for grade 2 and 1.70 (95 \% CI: 1.28-2.26) for grade 3 and above, showing no significant increase in $R R$ in grade 2 (Fig. 4). On the other hand, for UACR-based grades, there was a significant increase in $R R$ for cardiovascular events in both grade 2 (RR: 1.41; 95 \% CI: 1.19-1.66) and grade 3 and above (RR: 1.76; 95 \% CI: 1.37-2.28).

\section{Reclassification analysis}

Risk stratification capacities for cardiovascular events for dipstick-based risk grades (low risk (grade 1), intermediate risk (grade 2), high risk (grades 3 and 4)) vs. UACRbased grades derived from the reclassification table are shown in Fig. 5 . In the events group $(n=708), 80 \%$ were categorized as low risk by the dipstick-based model, compared with $51 \%$ by the UACR-based risk grade. In addition, according to dipstick-based CKD classifications, prevalence of CKD was only $20 \%$ in the event group (grade $\geq 2$ ), whereas with UACR-based CKD classification, prevalence of CKD was $49 \%$. 


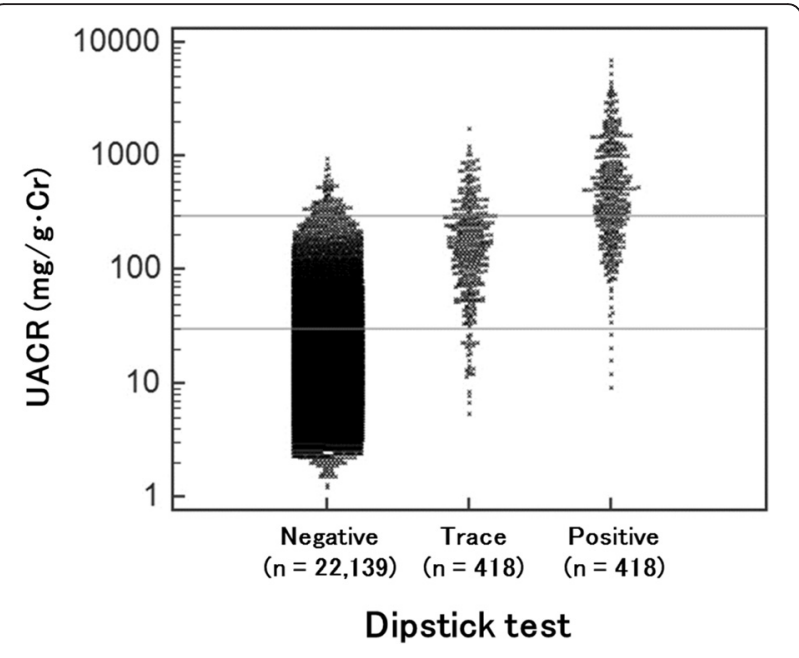

Fig. 3 Multiple comparison graph of UACR and dipstick tests

These results suggest that when the UACR level was used rather than dipstick testing for heat map risk grading, overall predictive ability for cardiovascular events significantly improved $(\mathrm{NRI}=0.232, \quad p<0.001 ; \quad \mathrm{IDI}=$ $0.0024, p<0.001)$.

\section{Discussion}

Results of this study suggest that the cardiovascular predictive performance for CKD risk grading based on UACR plus reduced eGFR (UACR-based risk grading) was superior to that of dipstick testing plus reduced eGFR (dipstick-based risk grading) in the general population.

In addition to generating a high degree of risk for the development of end-stage renal disease, CKD is also an important risk factor for cardiovascular events such as

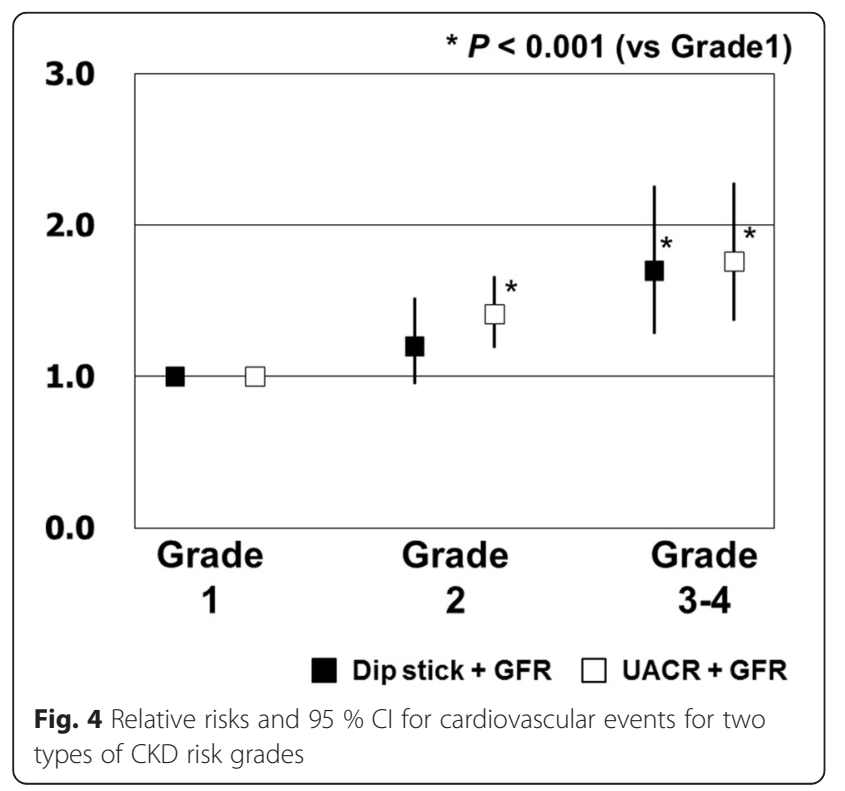

atherosclerotic coronary heart disease, stroke, and allcause mortality $[1,2,4]$. CKD is therefore a more important risk factor for cardiovascular disease than diabetes [18]. Several studies have reported that the number of patients experiencing cardiovascular events and cardiovascular death is greater than the number receiving dialysis $[1,3]$. It has been determined that the coexistence of CKD in patients with cardiac disorders is one of the important independent prognostic markers $[19,20]$. In view of this, it is recommended that patients at high risk of a cardiovascular event should have the risk managed early on, and should undergo screening to determine both GFR and urine albumin [21].

UACR measurement is somewhat costly compared with dipstick urinalysis, especially in mass screening settings. Therefore, although dipstick testing has low sensitivity for renal dysfunction [22], this form of testing is frequently used in general population studies because of its simplicity and low cost. Few studies have reported on the relationship between UACR and dipstick testing in large-scale general population samples of more than 20 000 participants. Konta et al. reported that, in the general population $(n=\sim 2300)$, cases with negative dipstick test results sometimes showed UACR $\geq 30 \mathrm{mg} / \mathrm{g} \mathrm{Cr}$, and that even trace levels of proteinuria detected by dipstick testing often showed UACR $\geq 300 \mathrm{mg} / \mathrm{g} \mathrm{Cr}$ in diabetic or elderly patients [13]. Indeed, the degree of coincidence between UACR and dipstick testing was suboptimal in the current study's population (Fig. 3).

To the best of the authors' knowledge, no reports have directly compared the two methodologies (UACR-based risk grading or dipstick-based risk grading) to determine the better cardiovascular risk predictor in screening settings for the general population. The current study suggests that risk grading based on UACR is superior for predicting the development of cardiovascular events compared with dipstick-based grading (Fig. 5).

Prevalence of UACR $>30 \mathrm{mg} / \mathrm{g} \mathrm{Cr}$ was found to be $25 \%$ in the current study, a rate clearly higher than those found in populations in North America (NHANES III: 9 \%) [23], Europe (PREVEND study: $7 \%$ ) [24], and Australia (AusDiab Kidney study: 6 \%) [25]. The reason for the high prevalence of significant albuminuria in our population remains unknown. It may be, however, because of the higher age of our study cohort, as participants under 40 years of age were excluded. The cohort also included a high proportion of women (65\%). As women excrete less $\mathrm{Cr}$ per day than men, the urine albumin concentration corrected to the urine $\mathrm{Cr}$ concentration may have been calculated to be relatively high, and therefore easily exceeded the threshold of microalbuminuria of $>30 \mathrm{mg} / \mathrm{g} \mathrm{Cr}$. It is possible that these factors contributed to the relatively high prevalence of UACRbased CKD in women in the current cohort. 


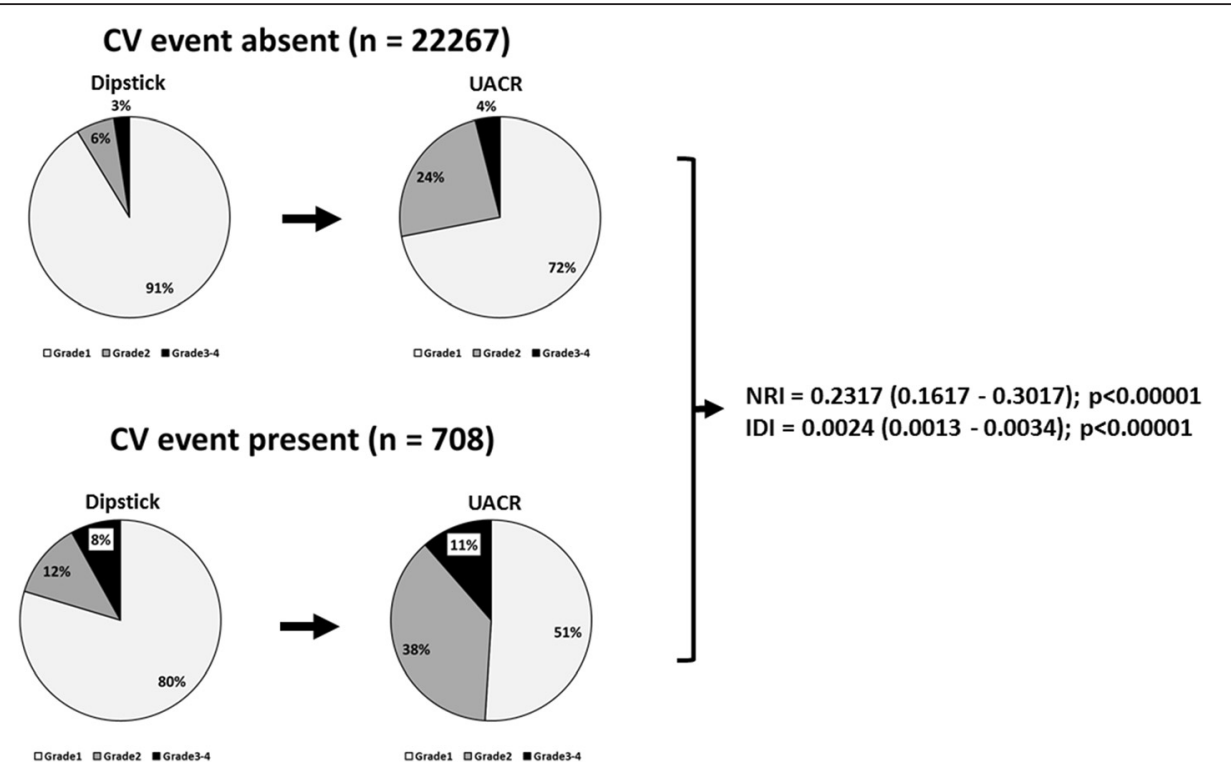

Fig. 5 Reclassification analysis. Changes in risk stratification capacity derived from reclassification tables in terms of dipstick-based CKD risk grades vs. UACR-based CKD risk grades. For comparison with the dipstick-based model, the percentage of risk grades 3-4 in the events group increased (from 20 to $49 \%$ ) in the UACR-based model. NRI: Net Reclassification Improvement; IDI: Integrated Discrimination Improvement

Given the high prevalence of microalbuminuria in this cohort, the high frequency of CKD was mainly because of the high incidence of UACR $\geq 30 \mathrm{mg} / \mathrm{g}$ Cr. Few reports directly describe the prevalence of CKD using UACR combined with the modern eGFR calculation (CKD-EPI) in a large number of participants. Therefore, the true prevalence of CKD as defined by UACR levels and reduced eGFR within the general population remains unclear. It seems unlikely that the high prevalence of CKD would be specific to the current population. Yamamoto et al. reported that $24 \%$ of patients with a negative dipstick test showed elevated UACR $\geq 30 \mathrm{mg} / \mathrm{g}$ $\mathrm{Cr}$ [26], which is almost the same as the value seen in the current study (22\%). Terawaki et al. reported that of the 241159 participants who received specific health examinations (mean age: 63 years, $39 \%$ men), mean eGFR was $76 \mathrm{~mL} / \mathrm{min} / 1.73 \mathrm{~m}^{2}$ [27], which again is almost the same as the current study. It therefore appears unlikely that renal function in the current cohort differs from that in populations from other regions of Japan.

Under the Japanese health insurance system, routine measurement of urine albumin is permitted for patients at high risk for cardiovascular disorders such as diabetes mellitus but not for other disorders. Microalbuminuria has been reported to be a risk factor for cardiovascular disease among non-diabetic and non-hypertensive patients [28]. It is possible that for patients with diabetes, hypertension, and obesity, cardiovascular risk assessment including albumin measurement for identification of CKD and more strict management of underlying disorders may improve prognosis. Hence, it may be beneficial to measure UACR to detect CKD even in populations at moderate cardiovascular risk, specifically diabetic, hypertensive, and obese people. As urine albumin measurement is relatively expensive compared with the cost of dipstick testing, further studies are required to identify the cost effectiveness of CKD screening using UACR for reducing cardiovascular events and mortality.

The current study has several strengths. To the best of the authors' knowledge, this study included the largest general population sample in whom UACR and urine dipstick testing has been performed. UACR was measured in fresh urine samples without long term freezing and repeated thawing. Cardiovascular events were captured prospectively according to previously determined standard epidemiological criteria and confirmed by research staff on medical chart reviews. Baseline data including clinical characteristics and biochemical data were determined well before the start of the follow-up study. However, despite the study's merits, some limitations must be considered when interpreting the results. First, measurement of urine albumin was based on a single measurement, which did not completely comply with the clinical requirement for multiple urine collections for establishing a diagnosis of CKD. Second, what effects prescribed drugs such as renin-angiotensin inhibitors have on cardiovascular events and urine albumin levels in the current population are not known. Third, the study employed only the CKD epidemiology collaboration (CKD-EPI) equation for eGFR and did not use other formulae, since there is no consensus on the best equation for eGFR in Asian populations. 


\section{Conclusion}

Although the prevalence of CKD in the general population was found to be higher when determined by eGFR and UACR compared with urine protein dipstick testing, the predictive ability for future cardiovascular events from UACR-based risk grading was superior to that of dipstick-based risk grading. Further studies including cost effectiveness analysis are needed to determine how best to utilize urine albumin measurement rather than dipstick testing to reduce the risk for cardiovascular events in mass screening settings.

\section{Availability of supporting data}

All data supporting the study is presented in the manuscript or available upon request from the corresponding author of this manuscript, Y Koeda.

\section{Ethics approval and consent to participate}

The study protocol was approved by the Ethics Committee of Iwate Medical University and conducted in accordance with the principles contained in the Declaration of Helsinki. All participants provided written informed consent.

\section{Abbreviations \\ BMI: body mass index; CKD: chronic kidney disease; CKD-EPI: Chronic Kidney Disease Epidemiology Collaboration; $\mathrm{Cr}$ : creatinine; HbA1c: hemoglobin A1c; IDI: Integrated Discrimination Improvement; KDIGO: Kidney Disease Improving Global Outcomes; NRI: Net Reclassification Improvement; RR: relative risk; UACR: urine albumin-creatinine ratio.}

\section{Competing interests}

The authors declare that they have no competing interests.

\section{Authors' contributions}

YK carried out data analysis and drafted the manuscript, FT revised the manuscript and performed myocardial infarction registration, TS revised the manuscript, MOhta performed health examination and assay, MOhsawa and KT participated the cohort coordination and checked death certificates, SM drafted the manuscript. YI and SO performed stroke registration, $\mathrm{KI}$ coordinated the cohort and advised study design, TO prepared the data set, KS advised the research design and the cohort coordination, $\mathrm{KO}$ performed stroke registration and chaired study meeting, $\mathrm{AO}$ designed the study and advised the cohort coordination, MN participated study designed and revised the manuscript. All authors read and approved the final manuscript.

\section{Acknowledgments}

We would like to thank Dr Yoshinori Kanaya, Dr Toshiaki Sakai, and Dr Osamu Nishiyama for their outstanding contributions to the registration of heart disease patients. The authors also thank the Iwate Stroke Registry Association and Northern Iwate Heart Registry Consortium for permission to use regional registry data for stroke and myocardial infarction.

\section{Funding}

This study was supported in part by grants-in-aid from the scientific research fund of the Ministry of Education, Science, and Culture of Japan (26461082), Tokyo, Japan; the Japan Arteriosclerosis Prevention Fund, Tokyo, Japan; and the Takeda Science Foundation (2012), Osaka, Japan.

\section{Author details}

'Division of Cardiology, Department of Internal Medicine, Iwate Medical University, 19-1 Uchimaru, Morioka, Iwate 020-8505, Japan. ${ }^{2}$ Iwate Health Service Association, Morioka, Japan. ${ }^{3}$ Department of Hygiene and Preventive Medicine, Iwate Medical University, Morioka, Japan. ${ }^{4}$ Department of
Neurosurgery, Iwate Medical University, Morioka, Japan. ${ }^{5}$ The Research Institute of Strategy for Prevention, Tokyo, Japan.

Received: 29 May 2015 Accepted: 9 May 2016

Published online: 12 May 2016

\section{References}

1. Go AS, Chertow GM, Fan D, McCulloch CE, Hsu CY. Chronic kidney disease and the risks of death, cardiovascular events, and hospitalization. N Engl J Med. 2004;351:1296-305.

2. Matsushita K, van der Velde M, Astor BC, Woodward M, Levey AS, de Jong PE, Coresh J, Gansevoort RT. Association of estimated glomerular filtration rate and albuminuria with all-cause and cardiovascular mortality in general population cohorts: a collaborative meta-analysis. Lancet. 2010;375:2073-81.

3. Keith DS, Nichols GA, Gullion CM, Brown JB, Smith DH. Longitudinal followup and outcomes among a population with chronic kidney disease in a large managed care organization. Arch Intern Med. 2004;164:659-63.

4. Levey AS, de Jong PE, Coresh J, El Nahas M, Astor BC, Matsushita K, Gansevoort RT, Kasiske BL, Eckardt KU. The definition, classification, and prognosis of chronic kidney disease: a KDIGO Controversies Conference report. Kidney Int. 2011;80:17-28.

5. Methven S, Traynor JP, Hair MD, St J, O'Reilly D, Deighan CJ, MacGregor MS. Stratifying risk in chronic kidney disease: an observational study of UK guidelines for measuring total proteinuria and albuminuria. QJM. 2011;104:663-70.

6. Johnson DW, Jones GR, Mathew TH, Ludlow MJ, Chadban SJ, Usherwood T, Polkinghorne K, Colagiuri S, Jerums G, Macisaac R, Martin H, Australasian Proteinuria Consensus Working Group. Chronic kidney disease and measurement of albuminuria or proteinuria: a position statement. Med J Aust. 2012;197:224-5.

7. Horio M. Development of evaluation of kidney function and classification of chronic kidney disease (CKD)-including CKD clinical practice guide 2012. Rinsho Byori. 2013;61:616-21 (In Japanese).

8. Ohsawa M, Itai K, Tanno K, Onoda T, Ogawa A, Nakamura M, Kuribayashi T, Yoshida Y, Kawamura K, Sasaki S, Sakata K, Okayama A. Int J Cardiol. 2009; 137:226-35.

9. Nakamura M, Onoda T, Itai K, Ohsawa M, Satou K, Sakai T, Segawa T, Sasaki J, Tonari Y, Hiramori K, Okayama A. Association between serum C-reactive protein levels and microalbuminuria: a population-based cross-sectional study in northern Iwate. Japan Intern Med. 2004;43:919-25.

10. Ohsawa M, Tanno K, Itai K, Turin TC, Okamura T, Ogawa A, Ogasawara K, Fujioka T, Onoda T, Yoshida Y, Omama S, Ishibashi Y, Nakamura M, Makita S, Tanaka F, Kuribayashi T, Koyama T, Sakata K, Okayama A. Comparison of predictability of future cardiovascular events between chronic kidney disease (CKD) grade based on CKD epidemiology collaboration equation and that based on modification of diet in renal disease equation in the Japanese general population-Iwate KENCO Study. Circ J. 2013;77:1315-25.

11. Horio M, Imai E, Yasuda Y, Watanabe T, Matsuo S. Modification of the CKD epidemiology collaboration (CKD-EPI) equation for Japanese: accuracy and use for population estimates. Am J Kidney Dis. 2010;56:32-8.

12. KDIGO. Chapter 1: Definition and classification of CKD. Kidney Int Suppl. 2013;3:19. http://www.kdigo.org/clinical_practice_guidelines/pdf/CKD/ KDIGO_2012_CKD_GL.pdf (Accessed on Nov 04, 2013).

13. Konta T, Hao Z, Takasaki S, Abiko H, Ishikawa M, Takahashi T, Ikeda A, Ichikawa K, Kato T, Kawata S, Kubota I. Clinical utility of trace proteinuria for microalbuminuria screening in the general population. Clin Exp Nephrol. 2007:11:51-5.

14. White SL, Yu R, Craig JC, Polkinghorne KR, Atkins RC, Chadban SJ. Diagnostic accuracy of urine dipsticks for detection of albuminuria in the general community. Am J Kidney Dis. 2011;58:19-28.

15. World Health Organization MONICA Project. Event Registration Data Component, MONICA Manual Version 1.1. Document for meeting of MONICA Principal Investigators. Geneva: World Health Organization; 1986.

16. Study Project of Monitoring System for Cardiovascular Disease commissioned by the Ministry of Health and Welfare. Manual for the Registry and Follow-up of Stroke (in Japanese). Osaka, Japan: National Cardiovascular Center, 1988.

17. Omama S, Yoshida Y, Ogasawara K, Ogawa A, Ishibashi Y, Ohsawa M, Tanno K, Onoda T, Itai K, Sakata K, Okayama A. Incidence rate of cerebrovascular diseases in northern Japan determined from the Iwate Stroke Registry with an inventory survey system. J Stroke Cerebrovasc Dis. 2013;22:e317-22. 
18. Tonelli M, Muntner P, Lloyd A, Manns BJ, Klarenbach S, Pannu N, James MT, Hemmelgarn BR. Risk of coronary events in people with chronic kidney disease compared with those with diabetes: a population-level cohort study. Lancet. 2012:380:807-14.

19. Weiner DE, Tabatabai S, Tighiouart H, Elsayed E, Bansal N, Griffith J, Salem DN, Levey AS, Sarnak MJ. Cardiovascular outcomes and all-cause mortality: exploring the interaction between CKD and cardiovascular disease. Am 」 Kidney Dis. 2006;48:392-401.

20. Hamaguchi S, Tsuchihashi-Makaya M, Kinugawa S, Yokota T, Ide T, Takeshita A, Tsutsui H. Chronic kidney disease as an independent risk for long-term adverse outcomes in patients hospitalized with heart failure in Japan. Report from the Japanese Cardiac Registry of Heart Failure in Cardiology (JCARE-CARD). Circ J. 2009;73:1442-7.

21. Brosius 3rd FC, Hostetter TH, Kelepouris E, Mitsnefes MM, Moe SM, Moore MA, Pennathur S, Smith GL, Wilson PW. Detection of chronic kidney disease in patients with or at increased risk of cardiovascular disease: a science advisory from the American Heart Association Kidney And Cardiovascular Disease Council; the Councils on High Blood Pressure Research, Cardiovascular Disease in the Young, and Epidemiology and Prevention; and the Quality of Care and Outcomes Research Interdisciplinary Working Group: developed in collaboration with the National Kidney Foundation. Circulation. 2006;114:1083-7.

22. Nagrebetsky A, Jin J, Stevens R, James T, Adler A, Park P, Craven A, Shine B, Farmer A. Diagnostic accuracy of urine dipstick testing in screening for microalbuminuria in type 2 diabetes: a cohort study in primary care. Fam Pract. 2013;30:142-52.

23. Garg AX, Kiberd BA, Clark WF, Haynes RB, Clase CM. Albuminuria and renal insufficiency prevalence guides population screening: results from the NHANES III. Kidney Int. 2002;61:2165-75.

24. de Jong PE, Hillege HL, Pinto-Sietsma SJ, de Zeeuw D. Screening for microalbuminuria in the general population: a tool to detect subjects at risk for progressive renal failure in an early phase? Nephrol Dial Transplant. 2003; 18:10-3.

25. Atkins RC, Polkinghorne KR, Briganti EM, Shaw JE, Zimmet PZ, Chadban SJ. Prevalence of albuminuria in Australia: the AusDiab Kidney Study. Kidney Int. 2004;92:S22-4.

26. Yamamoto K, Yamamoto H, Yoshida K, Niwa K, Nishi Y, Mizuno A, Kuwabara M, Asano T, Sakoda K, Niinuma H, Nakahara F, Takeda K, Shindoh C, Komatsu Y. The total urine protein-to-creatinine ratio can predict the presence of microalbuminuria. PLoS One. 2014;10:9(3).

27. Terawaki H, Nakayama M, Asahi K, Kakamu T, Hayakawa T, Iseki K, Kimura K, Moriyama T, Yamagata K, Narita I, Fujimoto S, Tsuruya K, Konta T, Kondo M, Kurahashi I, Ohashi Y, Fukushima T, Watanabe T. Comparison of predictive value for first cardiovascular event between Japanese GFR equation and coefficient-modified CKD-EPI equation. Clin Exp Nephrol. 2014.

28. Arnlov J, Evans JC, Meigs JB, Wang TJ, Fox CS, Levy D, et al. Low-grade albuminuria and incidence of cardiovascular disease events in nonhypertensive and nondiabetic individuals: the Framingham Heart Study. Circulation. 2005;112:969-75.

\section{Submit your next manuscript to BioMed Central and we will help you at every step:}

- We accept pre-submission inquiries

- Our selector tool helps you to find the most relevant journal

- We provide round the clock customer support

- Convenient online submission

- Thorough peer review

- Inclusion in PubMed and all major indexing services

- Maximum visibility for your research

Submit your manuscript at www.biomedcentral.com/submit 